\title{
Structural State Detection Using Transmissibility and Non- negative Matrix Factorization
}

\author{
Tongqun Ren ${ }^{1,2}$, Meiling Hui ${ }^{2}$, Junsheng Liang ${ }^{* 1,2}$, Dazhi Wang ${ }^{2}$, Liang $\mathrm{He}^{2}$ and \\ Yonghang Chen $^{3}$ \\ ${ }^{1}$ Key Laboratory for Precision \& Non-traditional Machining of the Ministry of \\ Education, Dalian University of Technology, Dalian, 116024, China \\ ${ }^{2}$ Key Laboratory for Micro/Nano Technology and System of Liao Ning Province, \\ Dalian University of Technology, Dalian, 116024, China \\ ${ }^{3}$ School of Mechanical and Electrical Engineering, Wuyi University, Jiangmen, \\ 529020, China \\ ren_tq@dlut.edu.cn,hml8585660@sina.com,jsliang@dlut.edu.cn, \\ d.wag@dlut.edu.cn,hll8905@163.com,chenyonghang@126.com
}

\begin{abstract}
Transmissibility function (TF) was used in structural state detection which examined the change of the response-only vibration characteristics. The technology of signal correlation was utilized to obtain the TF between two testing points. Multiple TFs under different states were calculated as basic TFs and formed a state matrix. Subsequently, non-negative matrix factorization (NMF) was performed for the state matrix. Then both the basic and testing TFs were projected to the feature subspace derived from NMF so as to obtain the state feature index vectors, respectively. Finally, the Euclidean distance between state feature index vectors was defined as the state indicator. The experimental results indicated that this method can achieve better detection accuracy than that using magnitude indicator. Actually, a result with 100 percent correct detection was achieved when proper rule of dimensionality reduction was selected. This method is essentially a multivariate statistical process monitoring (MSPM) method. It is feasible in vibrationbased structural state detection in the situation where the excitation signals are unavailable or inaccessible.
\end{abstract}

Keywords: Transmissibility function, State detection, Response-only, Negative matrix factorization

\section{Introduction}

Structural state change will cause changes of structural physical properties such as mass, damping and stiffness. The changes in these physical properties will be reflected by the vibration characteristics. This has led to the development of vibration-based structural state detection methods, which examine the changes in the vibration characteristics of structures as the indicators of state [1]. Among the existing vibration-based method, to detect the structural state based on response-only data is more attractive for engineering structures, especially in the situations where the excitation force are unavailable or inaccessible.

The TF is a well-known linear system concept reflecting the intrinsic characteristics of structure. It does not depend on the excitation forces and needs neither modal identification nor analytic or numerical model of the structure. Thus, the TF was widely applied in the state detection in various engineering structure. For example, Zhang et al. used both translational and curvature transmissibility to detect damage on a composite beam, and also proposed magnitude and phase damage indicators [2]. Mao and Todd 
investigated two features computed from transmissibility measurement changes to quantify connection stiffness loss: root-mean-square error and dot-product difference [3]. Nuno et al., proposed a transmissibility damage indicator based on the response vector assurance criterion (RVAC) [4]. Beyond the experimental validation of using TF for state detection, the sensitivity of $\mathrm{TF}$ against structural mass and stiffness change was analytically derived and validated by numerical examples [5].

In real practice, noise always contaminates the measurements, which may lead to reduced sensitivity in the transmissibility as a state-sensitive feature. At this point, the statistics-based method should be more robust in dealing with a real implementation that is subject to background noise, measurement error, and other systematic uncertainties. Actually, the TFs are nonnegative multivariate vectors. Thus, the MSPM method should be more reasonable method such as principal component analysis (PCA), partial least squares (PLS) and Non-negative Matrix Factorization (NMF). Being different from PCA and PLS, the NMF proposed by Lee and Seung makes all of the decomposed components to be not negative in process of nonlinear dimensionality reduction [6]. This non-negative constraint reflects the essential characteristic of the data better. Wang et al. used NMF to reduce the dimensionality of time-frequency images data to diagnose fault for diesel valve trains [7]. Li et al. presented a fault detection method based on NMF for non-Gaussian processes, and the result of the simulation experiment indicated that the detection efficiency and accuracy of the NMF method is better that PCA method [8].

In this work, a structural state detection method using TF in conjunction with NMF was described. The structural state matrix was constructed by TFs served as the column vectors. Then NMF was employed to extract the state feature index vectors, and the Euclidean distance between the state feature index vectors was defined as the state indicator. The validity of this method was demonstrated by an actual experiment on the test platform of ballastless track.

\section{Materials and Methods}

\subsection{Transmissibility Function}

The TF is defined as the ratio of two response frequency spectra of like variables (motion response/motion input) $x_{k}$ and $x_{j}$. Suppose a n-degree-of-freedom (n-DOF) linear structure is excited by external force $f(t)=\left[f_{1}(t), f_{2}(t), \ldots, f_{\mathrm{n}}(t)\right]$. If the excitation force contains uniform spectral density (white-noise), such as ambient excitation, the fourier transform of $f_{k}(t)$ becomes a constant $C$. At this point, the acceleration TF between output DOF $i$ and reference-output DOF $j$ can be calculated as:

$$
T F_{i j}(\omega)=\frac{A_{i}(\omega)}{A_{j}(\omega)}=\frac{C \cdot \sum_{k=1}^{n} H_{i k}(\omega)}{C \cdot \sum_{k=1}^{n} H_{j k}(\omega)}=\frac{\sum_{k=1}^{n} H_{i k}(\omega)}{\sum_{k=1}^{n} H_{j k}(\omega)}
$$

where $A(\omega)$ is the frequency spectra of response acceleration signal, $H_{i k}(\omega)$ and $H_{j k}(\omega)$ are entries of the $n \times n$ frequency response function (FRF) matrix $H(\omega)$. If the external excitation force is only applied to the $k$-th DOF, i.e., $f(t)=\left[0,0, \ldots, f_{\mathrm{k}}(t), \ldots 0\right]$, Eq.(1) turns to:

$$
T F_{i j}(\omega)=\frac{A_{i}(\omega)}{A_{j}(\omega)}=\frac{H_{i k}(\omega)}{H_{j k}(\omega)}
$$

The TF defines how vibration (amplitude and phase) is transmitted between two testing points on structure. It carries complete information on the dynamic behavior of the structure. In the above two case, the excitation force does not participate in the calculation of TF, but just provides vibration energy. In other words, the TF only depends on the location of the excitation force rather than its property. According to Eq.(1) and Eq.(2), 
TF is a function of the FRF which is determined by the inherent characteristic of the structure. Therefore, TF can be utilized to deal with state detection.

In practice, the most common choice to estimate the TF is using an estimator given by Devriendt $\mathrm{C}$ et al. [9], which can be denoted as:

$$
T F_{i j}(\omega)=\frac{S_{i j}(\omega)}{S_{j j}(\omega)}
$$

where $S_{i j}(\omega)$ is the cross power spectra between the output $A_{i}(\omega)$ and the referenceoutput $A_{j}(\omega)$, and $S_{j j}(\omega)$ is the auto power spectra from the reference-output $A_{j}(\omega)$.

\subsection{Non-negative Matrix Factorization}

NMF is a multivariable analysis method defined as: In practical cases, the observations $V \in R_{+}{ }^{n \times m}$ will be approximated by $V \approx W H$, where $W \in R_{+}{ }^{n \times r}$ is the basis matrix and $H \in R_{+}^{r \times m}$ is the coefficient matrix.

Usually, $r$ is chosen less than $n$ or $m$, so that $W$ and $H$ are smaller than the original matrix $V$. The selection of $r$ generally meets the following condition:

$$
(m+n) r<m n
$$

The column vector in $V$ can be regarded as the weighted sum of that in $W$, while the weight coefficients, i.e., the corresponding elements of the column vector in $H$, are all positive. At this point, the NMF is actually an optimization problem that can be calculated using a multiplicative iterative algorithm according to a certain cost function $F$. The cost function is used to quantify the quality of the approximation, which can be usually constructed by using some measure of distance between two non-negative matrices. In this work, the measure was selected as the square of the Euclidean distance between $V$ and $W H$ denoted as [10]:

$$
F=\|V-W H\|_{F}^{2}=\sum_{i, j}\left(V_{i j}-(W H)_{i j}\right)^{2}
$$

The "multiplicative update rules" for NMF can be summarized as follows:

$$
H_{k j} \leftarrow H_{k j} \frac{\left(W^{T} V\right)_{k j}}{\left(W^{T} W H\right)_{k j}} ; W_{i k} \leftarrow W_{i k} \frac{\left(V H^{T}\right)_{i k}}{\left(W H H^{T}\right)_{i k}}
$$

\subsection{State Detection based on TF and NMF}

In application, multiple time-domain acceleration signals from two testing points are collected under different states, health or different type and degree of states. Then the corresponding TFs are calculated and stored as the basic TFs. Given an arbitrary pair of testing acceleration signals, the task is to judge what kind of state they belong to.

According to the principle of NMF, it is actually a process of dimensionality reduction. The matrix $W=\left[w_{1}, w_{2}, \ldots, w_{r}\right] \in R_{+}{ }^{n \times r}$ can be considered as a low dimensional feature subspace spanked by the original data set $V$, and $w_{i}$ is the base vector of the subspace. Therefore, the NMF is widely used in application of multivariate data process, such as face recognition. Here, we just treated the structural state detection as a problem that was congeneric with face recognition. The concrete process was summarized as follows:

Step 1: The state matrix $V \in R^{n \times m}$ is constructed using the modular vectors of the measured TFs (hereinafter referred to as TFs) under different states as column entries, where $n$ is the length of TF vectors, i.e., the number of the valid spectra lines that participate in the operation, and $m$ is the number of TFs.

Step 2: The NMF is performed for $V$ to obtain the matrix $W$. Then all the measured TFs are projected on $W$ so as to get a serial of projection vectors $s_{i}=W^{\mathrm{T}} \cdot \mathrm{TF}_{i}, i=1,2, \ldots, m$. 
Step 3: Given an arbitrary testing $\mathrm{TF}_{j}$, it is also projected on $W$ to get a projection vector $t_{j}=W^{\mathrm{T}} \cdot \mathrm{TF}_{j}$. The Euclidean distances between $t_{j}$ and $s_{i}$ are calculated.

Step 4: We judge the testing state being just the state that takes the minimal Euclidean distance between $t_{j}$ and $s_{i}$.

\section{Experiment and Discuss}

In this work, an experiment was carried out on the test platform of ballastless track with a length of 20 meters, which is built based on the design and construction standards of Harbin-Dalian (China) high-speed railway. Two accelerometers (PCB 353B03) were installed on the railhead of midspan with the interval of two groups of fasteners, as shown in Figure 1. A single excitation was applied on a fixed position at the left side of both the two sensors by a hammer with plastic hammerhead. Then the acceleration signals of output A and reference-output B were collected and utilized to calculate the TFs, where the DHDAS_5902 dynamic signal acquisition system is used. The state change of the track structure is obtained by loosening the fasteners between two testing points one after another. Thus, there were totally five states of the track structure, all fasteners tightened, one fastener loosened, two fasteners loosened, three fasteners loosened and four fasteners loosened.

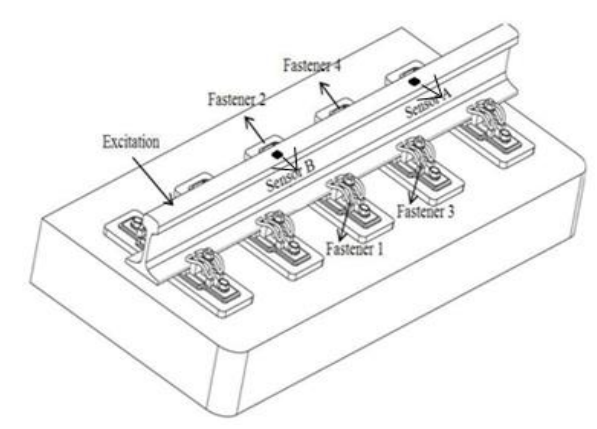

(a)

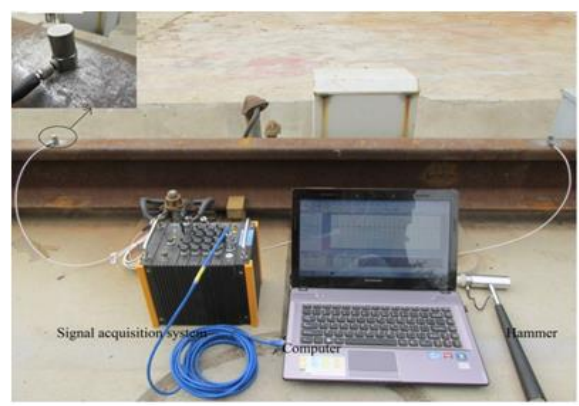

(b)

Figure 1. The Scene and Equipments of the Experiment (a) The Schematic Diagram, (b) The Experimental Scene and Equipments

Under each state, the track structure was excited 20 times that resulted in a total of 20 TFs, 15 of them were considered as the basic TFs and the remaining 5 TFs were considered as the testing TFs. The sampling frequency and sampling points are $10 \mathrm{kHz}$ and 1024, respectively, which meant the frequency resolution is $9.76 \mathrm{~Hz}$. All the 20 results of the TFs under state 1, i.e., state of all fasteners tightened were illustrated in Figure 2., where the valid frequency range was selected from 0.3 to $1.5 \mathrm{kHz}$ in order to reduce the influence of test-related uncertainty such as the low-frequency ambient noise. Thus, a state matrix $V \in R^{122 \times 75}$ was constructed. The average of the TFs under state 1 was also calculated and illustrated in Figure 3. Compared with Figure 2, there were visible differences among the average TFs under different states. 


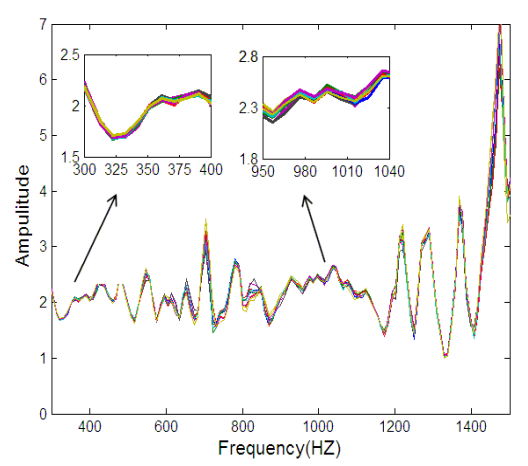

Figure 2. All the TFs of state 1

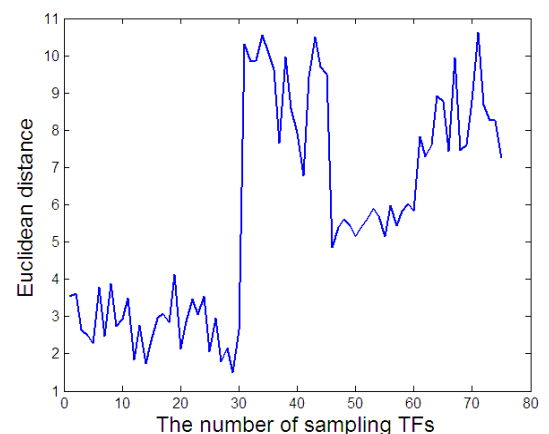

(a)

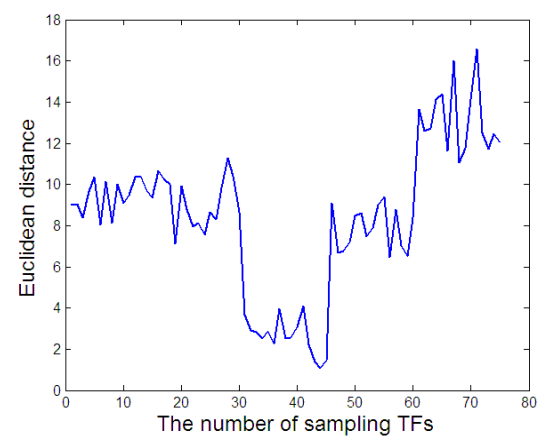

(c)

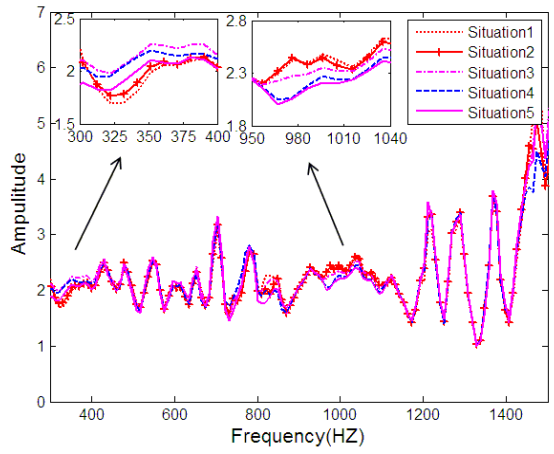

Figure 3. The average TF of state 1

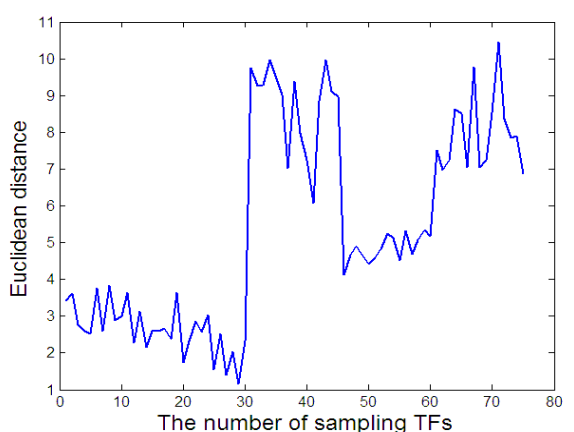

(b)

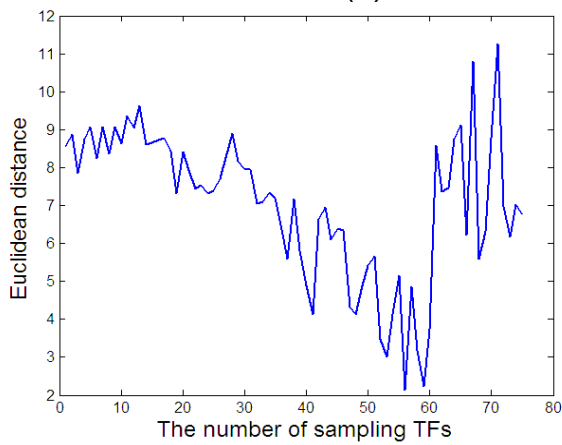

(d)

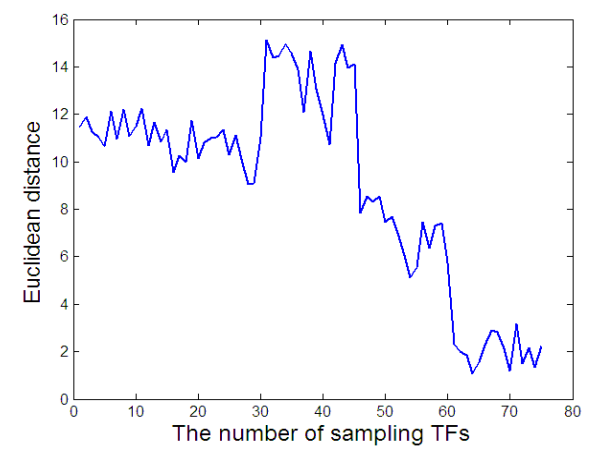

(e)

Figure 4. The Euclidean Distance Results with $r=10$ (a) Result of State 1, (b) Result of State 2, (c) Result of State 3, (d) Result of State 4, (e) Result of State 5

For each basic $\mathrm{TF}_{i}(i=1,2, \ldots, 75)$ and testing $\mathrm{TF}_{j}(j=1,2,3,4,5)$, the NMF was employed to extract the state feature index vectors by the method mentioned in Section 2.3, where 
the value of $r$ is selected as 10 . Then the Euclidean distance between state feature index vectors were calculated. The results of the last testing TF for each state were summarized in Figure 4.

Ideally, the former 15 results should be smaller than other ones if the testing state is state 1 , results of 16 to 30 should be smaller than other ones if the testing state is state 2 , and so on. However, the computed results will generate aliasing inevitably because of multiple factors such as computing error, tiny changes of incentive location and testrelated uncertainty. That was just the reason why multiple signals under one state were used, and the final decision was based on the minimal distance result. All the final detection results were listed in Table 1. For each testing TF, the first column recorded the serial number of basic TF that getting the minimal distance, and the second column recorded the result of state identification where symbol $\sqrt{ }$ meant correct identification and symbol $\times$ meant misidentification.

Table 1. The Final Detection Results with $r=10$

\begin{tabular}{c|c|c|c|c|c|c|c|c|c|c}
\hline \multirow{2}{*}{$\begin{array}{c}\text { State } \\
\text { No. }\end{array}$} & \multicolumn{9}{|c|}{ The serial number of matching basic TFs and detection results } \\
\cline { 2 - 11 } & \multicolumn{2}{|c|}{ Testing 1 } & \multicolumn{1}{|c|}{ Testing 2} & \multicolumn{2}{|c|}{ Testing 3 } & \multicolumn{2}{|c|}{ Testing 4 } & \multicolumn{2}{|c}{ Testing 5 } \\
\hline 1 & 15 & $\sqrt{ }$ & 3 & $\sqrt{ }$ & 3 & $\sqrt{ }$ & 27 & $\times$ & 29 & $\times$ \\
2 & 30 & $\sqrt{ }$ & 25 & $\sqrt{ }$ & 27 & $\sqrt{ }$ & 27 & $\sqrt{ }$ & 29 & $\sqrt{ }$ \\
3 & 44 & $\sqrt{ }$ & 44 & $\sqrt{ }$ & 43 & $\sqrt{ }$ & 44 & $\sqrt{ }$ & 44 & $\sqrt{ }$ \\
4 & 54 & $\sqrt{ }$ & 56 & $\sqrt{ }$ & 54 & $\sqrt{ }$ & 53 & $\sqrt{ }$ & 56 & $\sqrt{ }$ \\
5 & 63 & $\sqrt{ }$ & 70 & $\sqrt{ }$ & 71 & $\sqrt{ }$ & 71 & $\sqrt{ }$ & 64 & $\sqrt{ }$ \\
\hline
\end{tabular}

Similarly, the same process was performed again with $r=25$. The results of the last testing TF for each state were summarized in Fig.5, and the final detection results were listed in Table 2.

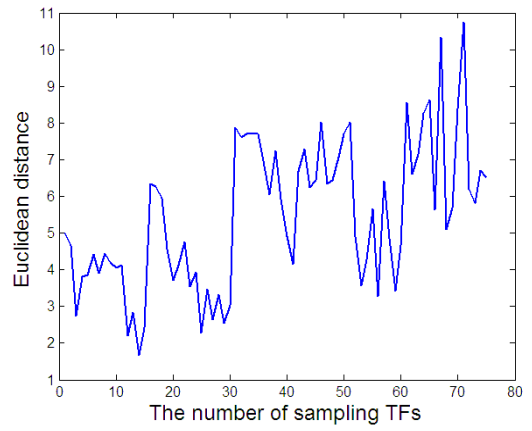

(a)

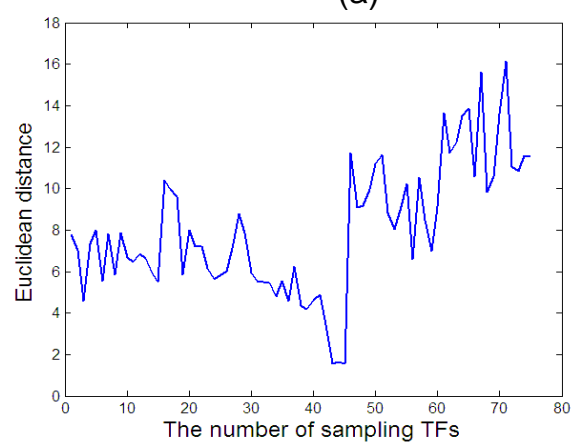

(c)

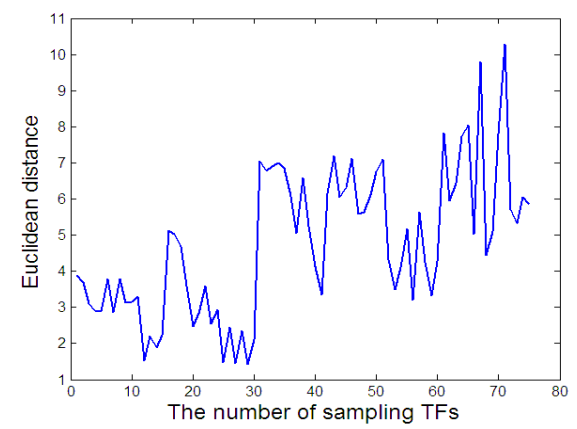

(b)

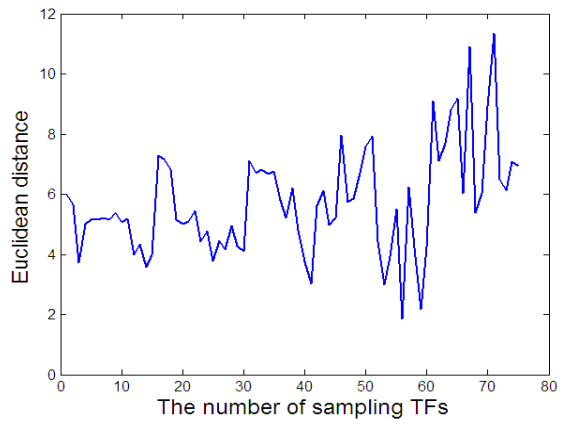

(d) 


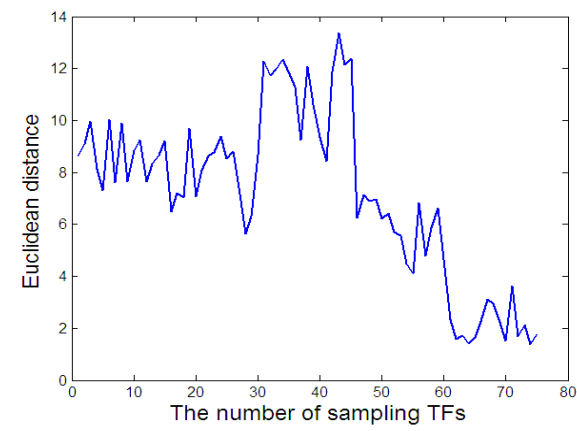

(e)

Figure 5. The Euclidean Distance Results with $r=25$ (a) Result of State 1, (b) Result of State 2, (c) Result of State 3, (d) Result of State 4, (e) Result of State 5

Table 2. The Final Detection Results with $r=25$

\begin{tabular}{c|c|c|c|c|c|c|c|c|c|c}
\hline \multirow{2}{*}{$\begin{array}{c}\text { State } \\
\text { No. }\end{array}$} & \multicolumn{9}{|c}{ The serial number of matching basic TFs and detection results } \\
& \multicolumn{10}{|c|}{ with $r=25$} \\
\cline { 2 - 10 } & \multicolumn{2}{|c|}{ Testing 1} & \multicolumn{2}{|c|}{ Testing 2} & \multicolumn{2}{|c|}{ Testing 3 } & \multicolumn{2}{|c|}{ Testing 4 } & \multicolumn{2}{|c}{ Testing 5 } \\
\hline 1 & 15 & $\sqrt{ }$ & 3 & $\sqrt{ }$ & 3 & $\sqrt{ }$ & 12 & $\sqrt{ }$ & 14 & $\sqrt{ }$ \\
2 & 25 & $\sqrt{ }$ & 25 & $\sqrt{ }$ & 27 & $\sqrt{ }$ & 27 & $\sqrt{ }$ & 29 & $\sqrt{ }$ \\
3 & 44 & $\sqrt{ }$ & 44 & $\sqrt{ }$ & 43 & $\sqrt{ }$ & 45 & $\sqrt{ }$ & 45 & $\sqrt{ }$ \\
4 & 54 & $\sqrt{ }$ & 56 & $\sqrt{ }$ & 54 & $\sqrt{ }$ & 53 & $\sqrt{ }$ & 56 & $\sqrt{ }$ \\
5 & 63 & $\sqrt{ }$ & 70 & $\sqrt{ }$ & 67 & $\sqrt{ }$ & 67 & $\sqrt{ }$ & 74 & $\sqrt{ }$ \\
\hline
\end{tabular}

The experimental results showed that different $r$ resulted in different detection result. As mentioned above, $W=\left[w_{1}, w_{2}, \ldots, w_{r}\right] \in R^{n \times r}$ is a low dimensional feature subspace spanked by the original data set $V$, and $w_{i}$ is the base vector of the subspace. Thus, the value of parameter $r$ determines the proximity between the original data and the data after dimensionality reduction. If the value of $r$ is too small, the data after dimensionality reduction is not sufficient to describe the original data. Some of useful information will be discarded inevitably after projection on the subspace, which will lead to fault detection. Conversely, if the value of $r$ is too large, more redundant information and noise will be reserved, the NMF method will lost its significance. Realistically speaking, we did not have an appropriate rule to select the optimal value of $r$, so far.

In order to further confirm the effectiveness of our method, the magnitude indicator proposed in Ref.[2] was also employed to deal with the state detection. For each state, the average of the former 15 TFs was considered as the standard TF in this state. Then the state indicator was calculated according to Eq.(7).

$$
D_{i}=\int_{0}^{\infty}\left|T F^{h}-T F_{i}{ }^{t}\right| d f / \int_{0}^{\infty}\left|T F^{h}\right| d f
$$

where $\mathrm{TF}^{h}$ means the standard $\mathrm{TF}$ in healthy state, $\mathrm{TF}_{i}^{t}$ means the $i$ th testing $\mathrm{TF}$. Here, state 1 was treated as the healthy state. Based on the basic TFs, the standard state indicator for each testing state was firstly calculated and denoted as $\mathrm{SD}_{i}, i=1,2,3,4,5$. Then the values of $\mathrm{D}_{i}$ for all the testing $\mathrm{TF}_{j} \mathrm{j}=1,2, \ldots, 25$, were calculated and illustrated in Figure 6 successively. We judged the testing $\mathrm{TF}_{j}$ belong to state $i$ if $\mathrm{SD}_{i-1} \leq \mathrm{D}_{i}<\mathrm{SD}_{i}$, where $\mathrm{SD}_{0}=0$. The final detection results were listed in Table 3, where the first column recorded the value of $\mathrm{D}_{i}$. It can be seen that more fault detection results were obtained than our method. 


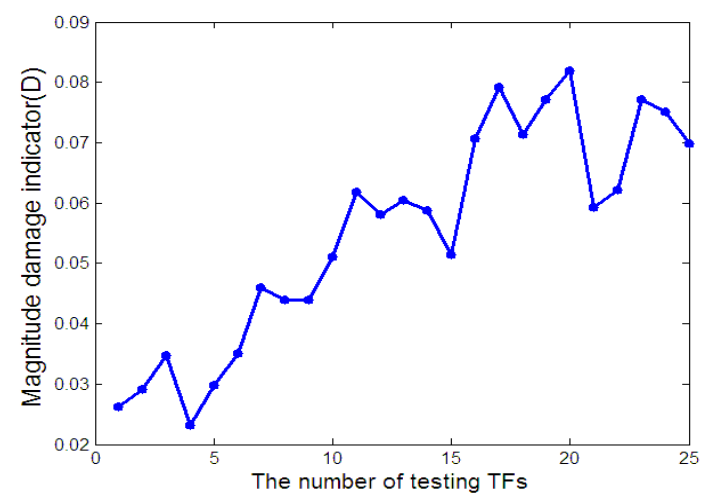

Figure 6. The values of $D_{i}$ using Magnitude Indicator

Table 3. The Final Detection Results using Magnitude Indicator

\begin{tabular}{|c|c|c|c|c|c|c|c|c|c|c|}
\hline \multirow{2}{*}{$\begin{array}{c}\text { Situatio } \\
n \\
\text { No. }\end{array}$} & \multicolumn{10}{|c|}{ The values of state indicators and detection results } \\
\hline & \multicolumn{2}{|c|}{ Testing 1} & \multicolumn{2}{|c|}{ Testing 2} & \multicolumn{2}{|c|}{ Testing 3} & \multicolumn{2}{|c|}{ Testing 4} & \multicolumn{2}{|c|}{ Testing 5} \\
\hline 1 & 0.026 & $\sqrt{ }$ & 00291 & $\sqrt{ }$ & 00347 & $\sqrt{ }$ & 0.0231 & $x$ & 0.029 & $\sqrt{ }$ \\
\hline & 3 & & & & & & & & 7 & \\
\hline 2 & 0.035 & $x$ & 00459 & ل & 00440 & 1 & 00439 & 1 & 0.051 & $\sqrt{1}$ \\
\hline & 0 & & ט. & & & & & & 1 & \\
\hline 3 & 0.061 & $\sqrt{ }$ & 00580 & y & 00605 & 1 & 0588 & 1 & 0.051 & $x$ \\
\hline & 9 & & & & 0.0000 & & 0.0000 & & 5 & \\
\hline 4 & 0.070 & $x$ & 00793 & $x$ & 0713 & $x$ & 00771 & $x$ & 0.082 & $x$ \\
\hline 4 & 7 & n & 0.070 & $\mathrm{n}_{\mathrm{s}}$ & (5) & ${ }_{2}$ & ודוד & $\hat{n}$ & 0 & \\
\hline 5 & 0.059 & × & 0 & 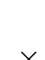 & 71 & d & 751 & 1 & 0.069 & 1 \\
\hline 5 & 3 & $x$ & 0.0021 & $x$ & ו & $\mathrm{v}$ & 15150.0 & $\mathrm{v}$ & 8 & $v$ \\
\hline
\end{tabular}

\section{Conclusions}

In this paper, a structural state detection method using techniques of TF and NMF was described. This method provided a solution to detect the presence of state change, but it can be further developed to detect both the presence and location of the state change by increasing the testing points and identifying the adjacent TFs successively. The availability of this method was demonstrated by an actual experiment on the test platform of ballastless track with an appropriate value of $r$ in NMF. The assignment rule for parameter $r$ is not discussed here and we need to do an in-depth research on it.

\section{Acknowledgments}

This work was supported by the National Natural Science Foundation of China (No. 51305064), the Scientific Research Project of Education Department of Liaoning Province Key Laboratory, China (No. L2012014), the Fundamental Research Funds for the Central Universities, China (No.DUT13LAB14), and Open Funds of Traction Power State Key Laboratory, Southwest Jiaotong University, China (No. TPL1309). 


\section{References}

[1] W. Fan and P. Z. Qiao, "Vibration-based Damage Identification Methods: A Review and Comparative Study", Structural Health Monitoring, vol.10, no.1, (2011), pp.83-111.

[2] H. Zhang, M. J. Schulz and F. Ferguson, "Structural health monitoring using transmittance functions", Mechanical Systems and Signal Processing, vol.13, no.5, (1999), pp.765-787.

[3] Z. Mao and M. Todd, "A structural transmissibility measurements-based approach for system damage detection", Proceedings of SPIE-The International Society for Optical Engineering, San Diego, CA, USA, (2010), v 7650, n PART1.

[4] Nuno M.M. Maia, Raquel A. B. Almeida, António P.V. Urgueira and Rui P.C. Sampaio, "Damage detection and quantification using transmissibility", Mechanical Systems and Signal Processing, vol.25, no.7, (2011), pp.2475-2483.

[5] D. P. Zhu, X. H. Yi and Y. Wang, "Sensitivity Analysis of Transmissibility Functions for Structural Damage Detection", Proceedings of SPIE-Nondestructive Characterization for Composite Materials, Aerospace Engineering, Civil Infrastructure, and Homeland Security, San Diego, CA, USA, (2011), Vol. 7983.

[6] D. D. Lee, H. S. Seung, "Learning the parts of object by non-negative matrix factorization", Nature, vol.401, no.6755, (1999), pp.788-791.

[7] Q. H. Wang, Y. Y. Zhang, L. Cai and Y. S. Zhu, "Fault diagnosis for diesel valve trains based on nonnegative matrix factorization and neural network ensemble", Mechanical Systems and Signal Processing, vol.23, no.5, (2009), pp.1683-1695.

[8] X. B. Li, Y. P. Yang and W. D. Zhang, "Fault detection method for non-Gaussian processed based on non-negative matrix factorization", Asia-Pacific Journal of Chemical Engineering, vol.8, no.3, (2013), pp.362-370.

[9] C. Devriendt, G. Steenackers, G. D. Sitter and P. Guillaume, "From operating deflection shapes towards mode shapes using transmissibility measurements", Mechanical Systems and Signal Processing, vol.24, no.3, (2010), pp.665-677.

[10] D. D. Lee, H. S. Seung, "Algorithms for non-negative matrix factorization", 14th Annual Neural Information Processing Systems Conference, NIPS 2000, Denver, CO, USA, (2000).

\section{Authors}

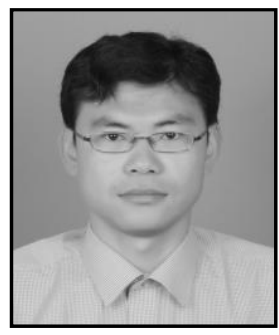

Tongqun Ren, he received his $\mathrm{PhD}$ degree in Precision Measuring Technology and Instruments at Tianjin University, China, in 2008. He is now an Associate Professor with the school of Mechanical Engineering at the Dalian University of Technology, China. His current research interests are in the fields of vibration detection, signal analysis, optical precision measurements, and instruments.

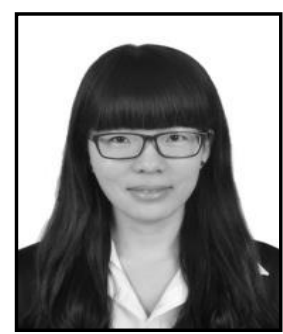

Meiling Hui, she received the BS degree from North China Institute of Aerospace, Langfang, China. Now she is a master student with School of mechnical engineering, Dalian University of Technology, China. Her current research interests include fault diagnosis and signal analysis.

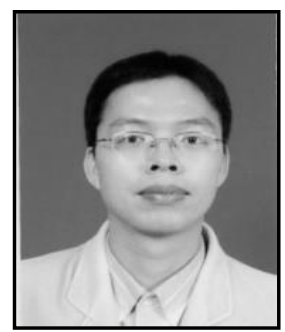

Junsheng Liang, he received his $\mathrm{PhD}$ degree in Mechanical and Electronic Engineering from Dalian University of Technology, China, in 2007. He is now an Associate Professor in School of Mechanical Engineering, Dalian University of Technology, China. His research interests include technology of measurement and control, micro-and nano-electromechanical systems and structural health monitoring. 


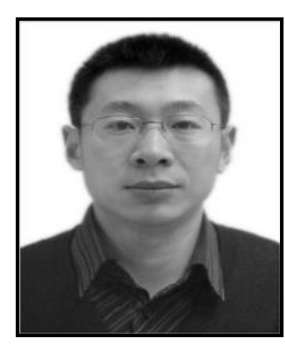

Dazhi Wang, he received his $\mathrm{PhD}$ degree in materials science from Queen Mary University of London, UK, in 2006. He is now a Professor in School of Mechanical Engineering, Dalian University of Technology, China. His current research interests are piezoelectric microsystems and structural health monitoring.

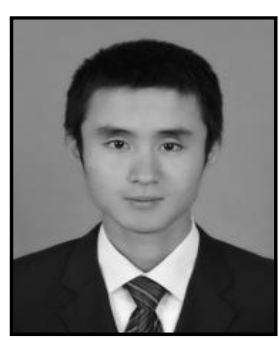

Liang He, he received the BS degree from Shandong University of Technology, China, in 2012. Now he is a master student with School of Mechanical Engineering, Dalian University of Technology, China. His current research interests include fault diagnosis and signal analysis.

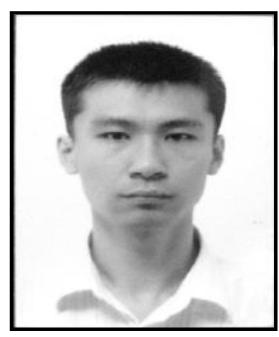

Yonghang Chen, he received the ME degree in industrial design from Tianjin University, China, in 2009. Now he is an engineer with School of Mechanical and Electrical Engineering, Wuyi University, China. His current research interests include industrial design and fault diagnosis. 